\title{
Visualization of Sub-atomic Movements in Nanostructures
}

\author{
Tongjun Liu*, Jun-Yu Ou*, Eric Plum*, Kevin F. MacDonald*, Nikolay I. Zheludev ${ }^{* \dagger}$ \\ * Optoelectronics Research Centre and Centre for Photonic Metamaterials, \\ University of Southampton, Highfield, Southampton, SO17 1BJ, UK
}

+ Centre for Disruptive Photonic Technologies, School of Physical and Mathematical Sciences and The Photonics Institute, Nanyang Technological University, Singapore, 637378, Singapore

\section{Noise Equivalent Displacement (NED)}

The NED level in HMV-SEM is determined largely by the Poisson statistics of electrons incident on the SE detector, whereby the standard deviation of shot noise is

$$
\sqrt{N}=\sqrt{I_{S E} \tau / e}
$$

$N$ being the number of electrons detected in time interval $\tau$.

The SE current gradient can be estimated as

$$
\nabla I_{S E}(\boldsymbol{r}) \sim \Delta I_{S E} / \rho
$$

where $\Delta I_{S E}$ is the change in SE current over a distance equal to the incident electron beam spot size $\rho$.

In measuring the amplitude of displacement $a$ by detecting secondary electron current variation, the number of electrons detected in time $\tau$ is then

$$
N=a\left(\Delta I_{S E} / \rho\right)(\tau / e) .
$$

A unitary signal-to-noise ratio (SNR) will be achieved when $N / \sqrt{N}=1$, which is to say when

$$
a \sim\left(\rho / \Delta I_{S E}\right) \sqrt{e / \tau} \sqrt{I_{S E}} .
$$

In practice (Fig. 2b), we record SE detector output voltage $V_{S E}=\Omega I_{S E}$ and evaluate the corresponding gradient

$$
\nabla V_{S E}(\boldsymbol{r}) \sim \Omega \Delta I_{S E} / \rho
$$

in which terms the measurable displacement with unitary SNR is

$$
a \sim\left(\rho / \Delta V_{S E}\right) \sqrt{e / \tau} \sqrt{V_{S E}} \sqrt{\Omega} .
$$

The constant of proportionality $\Omega$ is estimated at $\sim 3 \times 10^{10} \mathrm{Ohms}$, under an assumption that the full-scale $1.5 \mathrm{~V}$ value of $V_{S E}$ (Fig. $2 \mathrm{~b}$ ) is associated with a maximum current $I_{S E}$ of order 50 pA (from an incident beam current of $86 \mathrm{pA}$; a SE yield of 0.6 , typical for gold at $5 \mathrm{keV}$ [ref. ${ }^{1}$ ]; and SE collection efficiency approaching $100 \%$ for an SEM in a short working distance immersion lens configuration ${ }^{2}$ ). Thus, at the point marked by an asterisk in Fig. 2b, where $V_{S E}$ 
$\sim 1 \mathrm{~V}$ and $\Delta V_{S E} / \rho \sim 60 \mathrm{mV} / \mathrm{nm}$, we may expect to measure a displacement as small as $a \sim 1.2 \mathrm{pm}$ with a one second integration time.

In the driven motion mapping examples of Figs. 3 and 4, where integration time is reduced to $0.1 \mathrm{~ms}$, the expected NED level rises accordingly to $\sim 120 \mathrm{pm} / \mathrm{Hz}^{1 / 2}$.

\section{Electron beam-induced heating}

For the purpose of estimating electron beam-induced temperature change, we consider:

- a free-standing bilayer beam of $50 \mathrm{~nm}$ gold on $50 \mathrm{~nm}$ silicon nitride, with length $L=22 \mu \mathrm{m}$ and width $w=500 \mathrm{~nm}$, in vacuum;

- an electron beam with acceleration voltage $V=5 \mathrm{kV}$ and a beam current $I=86 \mathrm{pA}$ incident on the gold side of the beam, as used in experiments.

Monte Carlo modelling 5 shows that $>99 \%$ of electrons are stopped within the $100 \mathrm{~nm}$ (gold plus silicon nitride) thickness of the beam at this low electron energy. In keeping with prior works ${ }^{6}$ we assume that around $2.5 \%$ of incident electron beam power is absorbed as heat $H=0.025 I V \sim 10 \mathrm{nW}$, predominantly in the gold layer.

The temperature change $\delta T$ over the entire beam is related to the heat loss rate by Fourier's law $H=-\kappa A \delta T / L$, where $\kappa$ is thermal conductivity and $A=w t$ is cross-sectional area, $t$ being thickness. The thermal conductivity of gold $\left(300 \mathrm{Wm}^{-1} \mathrm{~K}^{-1}\right)$ is much larger than that of silicon nitride $\left(20 \mathrm{Wm}^{-1} \mathrm{~K}^{-1}\right)$ so the presence of the nitride layer is ignored for the purposes of this estimate (whereby $t=50 \mathrm{~nm}$ ), giving $\delta T \sim 29 \mathrm{mK}$.

Finite element modelling (the heat transfer module in COMSOL Multiphysics) gives a very similar result. Herein we assume:

- the presence of both layers, with specific heat capacities at constant pressure of 129 and $700 \mathrm{Jkg}^{-1} \mathrm{~K}^{-1}$ respectively for gold and silicon nitride;

- that the beam has an initial temperature $T=300 \mathrm{~K}$ and that one end (i.e. the anchored end of the cantilever) is attached to a thermostat at $300 \mathrm{~K}$;

- a $10 \mathrm{nW}$ point source of heat at one corner of the gold layer at the free end of the beam (i.e. the cantilever tip, as in the point measurement of Brownian motion presented in Fig. 2).

Under these conditions, the temperature at the opposite gold corner of the cantilever tip increases by $\delta T \sim 27 \mathrm{mK}$.

Root mean square thermal displacement is proportional to the square root of temperature, so the relative change resulting from a temperature change $\delta T$ is given by $\sqrt{1+\delta T / T}-1$. At $\sim 5 \times 10^{-5}$ in the present case (i.e. not more than a few tens of femtometers against the measured $\sim 1 \mathrm{~nm}$ thermal motion RMS cantilever tip displacements), this contribution is negligible.

In the general case, the magnitude of this perturbation will be a function of target material composition and geometry (i.e., electron interaction, thermal and mechanical properties), scaling with both electron energy and beam current. For example, in the present case, at $V=30 \mathrm{kV}$ (the maximum possible in many SEM systems) and $I=1 \mathrm{nA}$ (a relatively high value in SEM applications; $>10 \times$ higher than used in experiments), the relative change in cantilever RMS displacement amplitude due to electron beam-induced heating would reach $\sim 3 \times 10^{-3}-\mathrm{a} \sim \mathrm{pm}$ level comparable to the detection noise floor (NED, above), still two orders of magnitude smaller than measured thermal motion tip displacements. 


\section{Electron-to-cantilever momentum transfer}

A non-relativistic electron of energy $E$ has a velocity $v=\sqrt{2 E / m_{e}}$ and momentum $P=$ $m_{e} v$, where $m_{e}$ is the electron mass. ( $v / c \sim 0.1$ in the present case, so relativistic corrections can be ignored.) An electron beam of current $I$ contains $I / e$ electrons per second, where $e$ is the electronic charge, and may thus impart a maximum force $F=\frac{I}{e} P=\frac{I}{e} \sqrt{2 E m_{e}}$. This amounts to $\sim 2 \times 10^{-14} \mathrm{~N}$ in the present case.

From finite element modelling, the spring constant of a $22 \mu \mathrm{m}$ long gold-coated silicon nitride cantilever as studied here is $0.56 \mathrm{~N} / \mathrm{m}$, meaning that it would be deflected by not more than $\sim 35 \mathrm{fm}$ as a consequence of electron bombardment at the tip. This represents a negligible perturbation against the measured nanometric RMS cantilever tip displacements.

Again, in the general case, the magnitude of this perturbation will be a function of target material composition and geometry, scaling with both electron energy and beam current. In the present case, at $V=30 \mathrm{kV}$ and $I=1 \mathrm{nA}$, electron beam-induced cantilever tip displacement would reach $\sim 1 \mathrm{pm}$ - once more, a level comparable to the detection noise floor (NED, above) and still two orders of magnitude smaller than measured thermal motion displacement amplitudes.

\section{Cantilever resonance frequencies}

Cantilever mechanical properties are simulated using the structural mechanics module in COMSOL Multiphysics (finite element method). A rectangular $100 \mathrm{~nm}$ thick (50 $\mathrm{nm}$ each of silicon nitride and gold) $\times 500 \mathrm{~nm}$ wide cross-section is assumed. Densities of silicon nitride and gold are taken to be $\rho_{\mathrm{Si} 3 \mathrm{~N} 4}=3,100$ and $\rho_{\mathrm{Au}}=19,300 \mathrm{~kg} / \mathrm{m}^{3}$; and Young's moduli $E_{\mathrm{Si} 3 \mathrm{~N} 4}=$ $250, E_{\mathrm{Au}}=70 \mathrm{GPa}$. From this model, the three lowest Eigenfrequencies of a $22 \mu \mathrm{m}$ long cantilever are those of the

- fundamental out-of-plane flexural mode at $111 \mathrm{kHz}$;

- fundamental in-plane flexural mode at $518 \mathrm{kHz}$;

- $\quad$ and the $2^{\text {nd }}$-order out-of-plane flexural mode at $695 \mathrm{kHz}$.

These correlate well with measured resonance frequencies (Fig. 2) - small discrepancies being accounted for by manufacturing imperfections and internal stresses within/between the silicon nitride and gold layers ${ }^{7}$ (which also account for the slightly out-of-plane equilibrium position of the cantilever).

\section{Effective mass}

The effective mass of an oscillator $m_{\text {eff,n }}$ relates its potential energy $E_{p}$ to its $n^{\text {th }}$ Eigenfrequency $f_{n}$ and maximum displacement $d_{n}^{*}$ through the expression

$$
E_{p}=1 / 2 m_{\mathrm{eff}, n}\left(2 \pi f_{n}\right)^{2}\left|d_{n}^{*}\right|^{2}
$$

Potential energy can be determined through a volume integral

$$
E_{p}=1 / 2\left(2 \pi f_{n}\right)^{2} \int \rho(r)\left|d_{n}(r)\right|^{2} d V
$$


where $d_{n}(r)$ is the spatial displacement distribution ('shape') of the $n^{\text {th }}$ mode and $\rho(r)$ is the spatial density distribution of the structure. Combining these two equations gives

$$
m_{\mathrm{eff}, n}=\int \rho(r)\left|\frac{d_{n}(r)}{d_{n}^{*}}\right|^{2} d V=\int \rho(r)\left|R_{n}(r)\right|^{2} d V
$$

where $R_{n}(r)$ is the normalized displacement at position $r$ for the $n^{\text {th }}$ mode.

In some cases ${ }^{3,4}$ (e.g. ideal cantilevers, strings, and rectangular membranes of uniform density distribution) the ratio of effective to physical mass $m_{\mathrm{eff}} / m$ assumes a single modeindependent value for the geometry. In others (doubly-clamped beams, circular membranes), there are well-established sets of mode-dependent values. In the general case, the above volume integral can be evaluated numerically, for example via finite element modelling.

\section{Displacement Calibration}

From Eq. (2), the frequency-locked SE signal at a given point is proportional to the dot product of vectors for displacement and SE signal gradient in the image plane. To quantitatively calibrate motion, one must determine the direction of motion (as projected to the image plane), so as to know the direction in which to evaluate the gradient from the static SE image. For simple geometric objects, such as the cantilevers considered here, we have good a priori knowledge of its vibrational modes - we know, for example, for the viewing angle chosen in Fig. 2, that small amplitude oscillations in both in- and out-of-plane modes will present as displacements perpendicular to the long edges of the cantilever at almost all points in the image where motion is detectable. This direction is parallel to within a few degrees to the image plane $y$ axis so we can very reasonably calibrate displacement measurements by evaluating gradient uniformly in that direction.

However, in general one may not know how an object moves and such information is not contained in the static image. But, based on an assumption that closely-spaced points should move in the in same way, i.e. should share a common displacement vector $\boldsymbol{R}$, except where they are on opposing sides of a boundary where one part moves relative to another, a local statistical best fit for displacement direction and magnitude can be derived from the combination of static (providing local gradient) and frequency-locked motion images, as follows:

- The image is divided into 'superpixel' domains. For example, in the case of the Fig. 3 cantilever these are chosen as groups of $5 \times 3$ pixels as illustrated in Fig. S1a. (In general, the shape and size of superpixel elements would be selected according to object geometry and image noise level.)

- Movement direction is evaluated for all possible directions - i.e. angles $\varphi$ from 0 to $180^{\circ}$, measure counterclockwise relative to the $+x$ direction, in suitably chosen steps;

- A 'first estimate' value of displacement amplitude $R_{\text {initial }}$ is calculated from the values of average gradient and average frequency-locked SE signal over the superpixel.

- For a range of possible displacement amplitudes from $0.5 R_{\text {initial }}$ to $2.0 R_{\text {initial }}$ and for the full range of possible movement directions, the 'predicted' frequency-locked SE signal is calculated (per Eq. 2) and compared with the actual, measured value.

- $\quad$ The mean square error $\left(I_{S E-\text { predicted }}-I_{S E-\text { measured }}\right)^{2}$ summed over the superpixel is evaluated as a function of displacement direction $\varphi$ and displacement amplitude $R$ 
(Fig. S1c). The combination giving the lowest mean square error is taken to be the actual image plane displacement vector.

For the cantilever arm considered here, a displacement direction $\varphi=94^{\circ}$ is obtained, in accordance with expectation that it should be perpendicular to the long edges of the beam, which (as viewed in Fig. S1a) are inclined at $\sim 4^{\circ}$ to the $x$-axis.

For the calibration of displacement amplitudes in Figs. 3 and 4, where variations in displacement direction between adjacent superpixels (or indeed over the whole image) are small if not negligible, the best-fit movement direction for each superpixel is applied identically to each of its constituent data points. For more complex structures, one may alternatively apply a superpixel 'neighborhood' to the evaluation of best-fit movement for each individual source image point (or subsets of points smaller than the superpixel), i.e. by applying best-fit movement only to the central point (or subset of points) and then offsetting the superpixel by one point (or one subset unit) to calculate for the next.

(a)

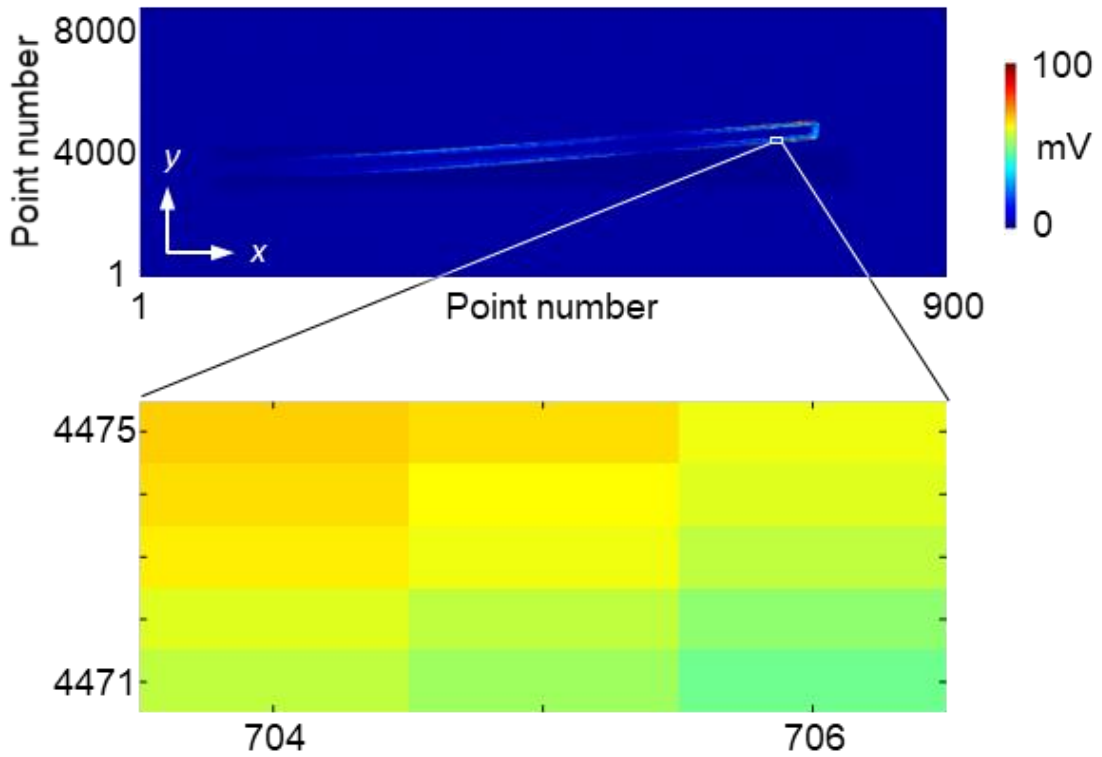

(b)

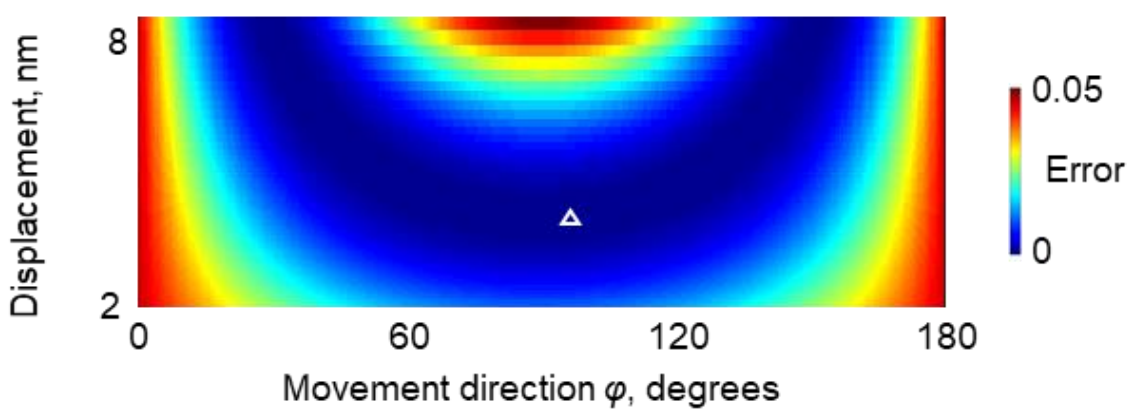

Fig. S1. Determination of displacement direction and amplitude. (a) $739.0 \mathrm{kHz} \mathrm{SE}$ signal amplitude image of a $22 \mu \mathrm{m}$ long gold-coated silicon nitride cantilever [as shown in Fig. $3 b$ but without the aspect ratio adjustment applied there] with enlarged detail of a sample $5 \times 3$ points superpixel located on one of the long edges of the beam. (b) Dependence on movement direction and predicted displacement amplitude of mean square error $\left(I_{S E-\text { predicted }}-I_{S E-\text { measured }}\right)^{2}$ summed over the superpixel, the value of which is minimized at the point denoted by the triangle symbol. 


\section{References}

1 Goldstein, J. I. et al. Scanning Electron Microscopy and X-Ray Microanalysis. $4^{\text {th }}$ edn, (Springer, 2018).

2 Müllerová, I. \& Konvalina, I. Collection of secondary electrons in scanning electron microscopes. J. Microsc. 236, 203-210 (2009).

3 Hauer, B. D., Doolin, C., Beach, K. S. D. \& Davis, J. P. A general procedure for thermomechanical calibration of nano/micro-mechanical resonators. Ann. Phys. - New York 339, 181-207 (2013).

$4 \mathrm{Kim}, \mathrm{J}$. E. On the equivalent mass-spring parameters and assumed mode of a cantilevered beam with a tip mass. J. Mech. Sci. Technol. 31, 1073-1078 (2017).

5 Drouin, D. et al. CASINO V2.42 - A Fast and Easy-to-use Modeling Tool for Scanning Electron Microscopy and Microanalysis Users. Scanning 29, 92-101 (2007).

6 Buks, E. \& Roukes, M. L. Stiction, adhesion energy, and the Casimir effect in micromechanical systems. Phys. Rev. B 63, 033402 (2001).

7 Karabalin, R. B., Villanueva, L. G., Matheny, M. H., Sader, J. E. \& Roukes, M. L. StressInduced Variations in the Stiffness of Micro- and Nanocantilever Beams. Phys. Rev. Lett. 108, 236101 (2012). 